\title{
Naphthazarin enhances ionizing radiation-induced cell cycle arrest and apoptosis in human breast cancer cells
}

\author{
MIN YOUNG KIM ${ }^{1,2^{*}}$, SEONG-JOON PARK ${ }^{1,3^{*}}$, JAE WOONG SHIM $^{1}$, \\ $\mathrm{KWANGMO} \mathrm{YANG}^{1,4,5}$, HO SUNG KANG ${ }^{2}$ and $\mathrm{KYU} \mathrm{HEO}^{1}$ \\ ${ }^{1}$ Research Center, Dongnam Institute of Radiological and Medical Science (DIRAMS), Busan 619-953; \\ ${ }^{2}$ Department of Molecular Biology, College of Natural Sciences, Pusan National University; \\ ${ }^{3}$ Department of Pharmacy, Pusan National University, Busan 609-735; ${ }^{4}$ Department of Radiation Oncology, \\ Dongnam Institute of Radiological and Medical Sciences, Busan 619-953; ${ }^{5}$ Department of Radiation Oncology, \\ Korea Institute of Radiological and Medical Sciences, Seoul 139-706, Republic of Korea
}

Received November 24, 2014; Accepted January 12, 2015

DOI: $10.3892 /$ ijo.2015.2857

\begin{abstract}
Naphthazarin (Naph, DHNQ, 5,8-dihydroxy1,4-naphthoquinone) is one of the naturally available 1,4-naphthoquinone derivatives that are well-known for their anti-inflammatory, antioxidant, antibacterial and antitumor cytotoxic effects in cancer cells. Herein, we investigated whether Naph has effects on cell cycle arrest and apoptosis in MCF-7 human breast cancer cells exposed to ionizing radiation (IR). Naph reduced the MCF-7 cell viability in a dose-dependent manner. We also found that Naph and/or IR increased the p53-dependent p21 (CIP/WAF1) promoter activity. Noteworthy, our ChIP assay results showed that Naph and IR combined treatment activated the p21 promoter via inhibition of binding of multi-domain proteins, DNMT1, UHRF1 and HDAC1. Apoptosis and cell cycle analyses demonstrated that Naph and IR combined treatment induced cell cycle arrest and apoptosis in MCF-7 cells. Herein, we showed that Naph treatment enhances IR-induced cell cycle arrest and death in MCF-7 human breast cancer cells through the p53-dependent p21 activation mechanism. These results suggest that Naph might sensitize breast cancer cells to radiotherapy by enhancing the p53-p21 mechanism activity.
\end{abstract}

Correspondence to: Dr Kyu Heo, Research Center, Dongnam Institute of Radiological and Medical Sciences (DIRAMS), Jwadong-gil 40, Gijang-gun, Busan 619-953, Republic of Korea

E-mail:khjhk33@gmail.com

Professor Ho Sung Kang, Department of Molecular Biology, College of Natural Sciences, Pusan National University, Busandaehak-ro63 beon-gil, Geumjeong-gu, Busan 609-735, Republic of Korea

E-mail: hspkang@ pusan.ac.kr

*Contributed equally

Key words: naphthazarin, ionizing radiation, cell cycle arrest, apoptosis

\section{Introduction}

Breast cancer is a major health problem in women worldwide and the main cause of cancer death among women. Many breast cancer patients fail conventional treatment strategies of chemotherapy, radiation and anti-estrogen therapy $(1,2)$. Therefore, the search for new and effective drugs to treat breast cancer is required. Research into the molecular pathway and biomarkers associated with the development of breast cancer is needed to find successful therapeutic approaches.

Plant-derived drugs play an increasingly important role in cancer therapy due to their low toxicity and high efficacy. Natural products derived from plants, such as camptothecin, vincristine, taxol, etoposide and paclitaxel, have received extensive attention as potential anticancer drugs. These bioactive phytochemicals are known to exert anticancer activity through different mechanisms, including immune activation, suppression of cell cycle progression and induction of apoptosis (3-11). Naphthazarin (Naph, DHNQ, 5,8-dihydroxy-1,4-naphthoquinone) is a naturally occurring 1,4-naphthoquinone derivative; a lipophilic red pigment like alkannin and shikonin. Naph derivatives are well-known for their anti-inflammatory, antioxidant, antibacterial antifungal and wound healing effects. They are also known for antitumor cytotoxic effects in cancer cells (12-16). The mechanism of antitumor cytotoxicity and the specific molecular target of Naph in cancer cells are yet to be established, but they probably involve the induction of differential expression of cell cycle regulators. Cell cycle progression is governed by cyclin-dependent kinases (CDKs) that are activated by cyclin binding and inhibited by CDK inhibitors (17,18). P21 is a CDK inhibitor (CKI) that plays a crucial role in arresting cellular growth, differentiation and apoptosis. P21 gene expression is induced by the p53 gene, thereby directly mediating p53-induced cell cycle arrest (19-24).

UHRF1 (Ubiquitin-like containing PHD and ring finger domains 1), also known as ICBP90 or Np95, is a multi-domain protein associated with cellular proliferation and epigenetic regulation. UHRF1 binds to methylated $\mathrm{CpG}$ dinucleotides and recruits the transcriptional repressors DNA methyltransferase 1 (DNMT1) and histone deacetylase 1 (HDAC1) to 
regulate gene expression (25-30). In addition, UHRF1 forms a complex with DNMT1/HDAC1 and binds to the promoter regions of tumor suppressor genes such as p16INK4A, p14ARF and $\mathrm{p} 21$ in cancer cells (31-36). In this study, we demonstrated that treatment with Naph and irradiation (IR) downregulates the expressions of DNMT1, UHRF1 and HDAC1, whereas it upregulates the expression of p21 in MCF-7 cells. Moreover, cell cycle arrest and apoptosis were significantly increased by combinatorial treatment of Naph and IR. Collectively, our results suggest that Naph might be an effective radiosensitizer and adjuvant therapy for breast cancer.

\section{Materials and methods}

Cell and culture conditions. Human breast cancer cell line, MCF-7, was obtained from the American Type Culture Collection (Rockville, MD, USA). Cells were maintained in DMEM (Welgene, Daegu, Korea) supplemented with $10 \%$ fetal bovine serum (FBS, HyClone, Logan, UT, USA) and 5\% antibiotic-antimycotic (Gibco, Grand Island, NY, USA). Cells were cultured at $37^{\circ} \mathrm{C}$ in a humidified atmosphere of $5 \% \mathrm{CO}_{2}$.

Drug treatment and IR exposure. Stock solutions of $10 \mathrm{mM}$ naphthazarin (Sigma-Aldrich, St. Louis, MO, USA) were dissolved in dimethyl sulfoxide (DMSO) and diluted in culture medium to the indicated final concentration for cell treatment. Cells were incubated at $37^{\circ} \mathrm{C}$ overnight and then treated with naphthazarin (Naph). After $2 \mathrm{~h}$, cells were exposed to gamma-rays from a $137 \mathrm{Cs} \gamma$-ray source (Eckert \& Ziegler, Berlin, Germany) at a dose rate of $2.6 \mathrm{~Gy} / \mathrm{min}$. Following IR at a $10 \mathrm{~Gy}$ dose, the cells were incubated under naphthazarin conditions for the indicated times.

Cell proliferation assay and cell morphology. Cell proliferation was assessed using the MTT colorimetric assay. MCF-7 cells $\left(2 \times 10^{5}\right.$ cells/well $)$ were seeded in 6 -well plates and incubated at $37^{\circ} \mathrm{C}$ overnight $(\mathrm{O} / \mathrm{N})$. After $24 \mathrm{~h}$ of culture, the medium was removed and replaced with experimental medium. Cells were pretreated with Naph (respective concentration) before $2 \mathrm{~h}$ and then exposed to IR at a $10 \mathrm{~Gy}$ dose for $24 \mathrm{~h}$. Subsequently, cells were washed twice with PBS and $5 \mathrm{mg} / \mathrm{ml}$ MTT in PBS was added to each well for $4 \mathrm{~h}$. After removal of the MTT solution, a solubilization solution (DMSO/EtOH, 1:1 ratio) was added to each well to dissolve the formazan crystals. The absorbance at $570 \mathrm{~nm}$ was measured using a Paradigm ${ }^{\mathrm{TM}}$ Detection Platform (Beckman Coulter, Inc., Fullerton, CA, USA). For investigation of morphological changes, Naph or/and IR treated cells were examined under an inverted light microscope (Nikon) after $48 \mathrm{~h}$.

RNA isolation and quantitative real-time PCR. Total RNA was isolated from the MCF-7 breast cancer cells using TRI-Solution (Bio Science Technology, Rockaway, NJ, USA) according to the manufacturer's protocol. The quantity of isolated RNA was measured using NanoDrop (Thermo Scientific, Rockford, IL, USA) and $1 \mu \mathrm{g}$ of RNA was reverse-transcribed using the iScript $^{\mathrm{TM}}$ cDNA synthesis kit (Bio-Rad). The following qPCR primers were used: sense HDAC1 5'-TGGAAATCTATCG CCCTCAC - 3 ' and antisense HDAC1 5'-TCTCTGCATCTGCT TGCTGT-3'; sense DNMT1 5'-GAGCTACCACGCAGAC
ATCA-3' and antisense DNMT1 5'-CGAGGAAGTAGAA GCGGTTG-3'; sense UHRF1 5'-CTGGGGGATGATTCT CTGAA-3' and antisense UHRF1 5'-CTCTTCCGTCTCA TGGGGT-3'; sense p21 5'-ATGGAACTTCGACTTTGTC ACC-3' and antisense p21 5'-AGGCACAAGGGTACAA GACAGT-3'; sense $\beta$-actin 5'-AGCGAGCATCCCCCAAA GTT-3' and antisense $\beta$-actin 5'-GGGCACGAAGGCTC ATCATT-3'.

Western blot analysis. Total cell lysates were loaded onto SDS-PAGE and transferred to PVDF (GE Healthcare Life Sciences, Piscataway, NJ, USA). The membranes were incubated overnight at $4^{\circ} \mathrm{C}$ with the primary antibodies. The following primary antibodies were used: anti-DNMT1 (Sigma-Aldrich), anti-UHRF1 (BD Bioscience), anti-HDAC1 (Abcam), anti-p53 (Santa Cruz Biotechnology Inc., Santa Cruz, CA, USA), anti-p21 (Abcam) and anti- $\beta$-actin (SigmaAldrich). On the following day, the membranes were washed for $10 \mathrm{~min}, 3$ times each, and incubated with the secondary antibody: polyclonal anti-rabbit antibody (Invitrogen, Carlsbad, CA, USA) or monoclonal anti-mouse antibody (Invitrogen). The immunoreactive proteins were detected using enhanced chemiluminescence (Thermo Scientific). Immunoblots were quantified using the ImageMaster densitometry program.

Chromatin immunoprecipitation (ChIP). ChIP assays were performed using the Magna ChIP kit (Millipore) according to the manufacturer's protocol. The following primers were used: sense p21 5'-TGGACTGGGCACTCTTGTCC -3' and antisense p21 5'-CA-GAGTAACAGGCTAAGGTT-3'. Anti-DNMT1 (Sigma-Aldrich), anti-UHRF1 (BD Biosciences), anti-HDAC1 (Abcam) and anti-p53 (Santa Cruz Biotechnology Inc.) antibodies were used to immunoprecipitate chromatin fragments.

Cell cycle analysis. Cells were treated with $1 \mu \mathrm{M}$ Naph or/and $10 \mathrm{~Gy}$ IR for $24 \mathrm{~h}$. The cells were trypsinized and resuspended in PBS. Then, cells were centrifuged and washed in PBS. Following fixation in cold $70 \%$ ethanol for $30 \mathrm{~min}$ at $4{ }^{\circ} \mathrm{C}$, the cells were stained with PI $(40 \mu \mathrm{g} / \mathrm{ml})$ and RNAse A $(50 \mu \mathrm{g} / \mathrm{ml})$ prior to analysis. The stained cells were subjected to cell cycle analysis using FACSAria (BD Biosciences).

Apoptosis analysis. The Annexin $\mathrm{V}$ analysis was carried out using the PE Annexin V apoptosis detection kit (BD Biosciences). After treatment of $1 \mu \mathrm{M}$ Naph or/and $10 \mathrm{~Gy}$, cells were washed in cold PBS and then resuspended in $1 \mathrm{X}$ binding buffer and incubated with PE Annexin V $(2.5 \mu \mathrm{g} / \mathrm{ml})$ conjugated primary antibody and 7-amino-actinomycin (7-AAD, $5 \mu$ l) for $15 \mathrm{~min}$ on ice. Following incubation, PI $(10 \mu \mathrm{g} / \mathrm{ml})$ was added to the suspension, and the cells were analyzed by FACSAria (BD Biosciences).

\section{Results}

Naph and IR inhibit proliferation of human MCF-7 breast cancer cells. Since Naph and/or ionizing radiation (IR) modulate cell proliferation or viability, we first examined the regulatory effect of Naph and IR on cell growth of MCF-7 cells. We treated MCF-7 cells with Naph for $48 \mathrm{~h}$ and cell viability was measured by the MTT assay. The viability of MCF-7 cells 

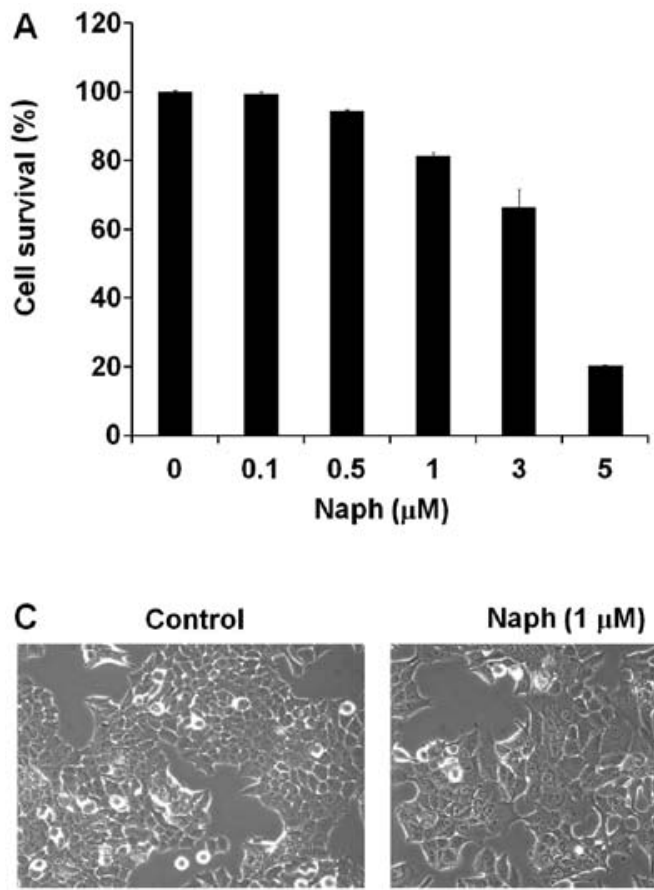
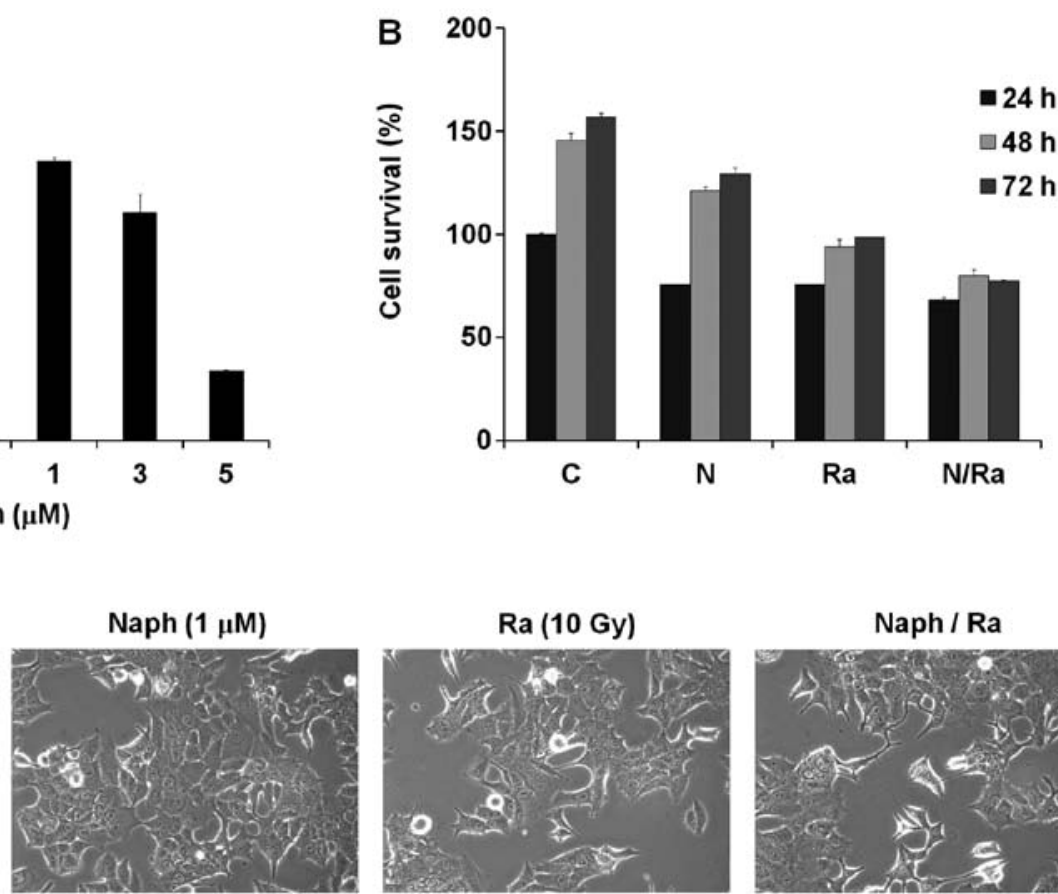

Figure 1. Cell viability and cell morphological changes induced by combinational treatment of Naph and IR in MCF-7 cells. (A) Comparative cell viability in the presence of Naph at the indicated concentration for $48 \mathrm{~h}$. (B) The results of MTT assay were assessed in Naph (1 $\mu \mathrm{M})$ and/or IR (10 Gy) treated cells at 24 , 48 and $72 \mathrm{~h}$. Graph represents the data (mean \pm standard deviation) from triplicate wells. The average absorbance values were plotted on the $y$-axis with each culture condition on the x-axis. C, untreated condition; N, Naph (1 $\mu \mathrm{M}) ; \mathrm{R}$, IR (10 Gy); NR, Naph (1 $\mu \mathrm{M})+$ IR (10 Gy). (C) Cell morphology was observed with phase contrast microscopy.

treated with Naph was decreased in a dose-dependent manner (Fig. 1A). We used the $1 \mu \mathrm{M}$ of Naph for further experiments to observe its synergistic effect with radiation. To investigate the synergistic effect of Naph and IR, MCF-7 cells were treated for 24, 48 and $72 \mathrm{~h}$ with Naph or $10 \mathrm{~Gy}$ of IR as a single treatment, or in combination. The results from the MTT assay showed that the combination of Naph and IR was more robust than that of Naph or IR single-treatment (Fig. 1B). MCF-7 cells typically exhibit a cobblestone-like appearance and tight cell-cell junction which is the characteristic of the epithelial phenotype $(37,38)$, but when the cells were treated with Naph and IR, morphological changes and less number of prominent cells were observed (Fig. 1C).

Naph and IR upregulate 21 expression and downregulate the expression of DNMT1, UHRF1 and HDAC1. Several studies have shown that the DNMT1/UHRF1/ HDAC1 complex negatively regulates the expression of $\mathrm{p} 21$, which inhibits cell proliferation (31-36). Since Naph and IR decrease MCF-7 cell proliferation, we first determined the expression levels of p21 and its corepressors, DNMT1, UHRF1 and HDAC1 after treatment with Naph. We treated the MCF-7 cells with Naph at a concentration of 1,3 and $5 \mu \mathrm{M}$ for $24 \mathrm{~h}$. The results of Real-time PCR indicated that Naph downregulated DNMT1, UHRF1 and HDAC1 mRNA expressions in a dose-dependent manner (Fig. 2A). In order to determine the combinatorial effect of Naph and IR on the expression of these genes, we treated the MCF-7 cells with Naph and IR under the indicated conditions. The MCF-7 cells were pre-incubated with Naph for $2 \mathrm{~h}$ prior to exposure to IR. The results showed that Naph significantly enhanced IR-induced p21 mRNA induction via decrease in DNMT1, UHRF1 and HDAC1 in MCF-7 cells (Fig. 2B). Since p21 is a downstream target of p53 which plays a key role in the regulation of cell cycle and apoptosis, we measured the protein levels of p53 and p21 together with levels of repressive factors including DNMT1, UHRF1 and HDAC1. In MCF-7 cells, the expression levels of p53 and its downstream $\mathrm{p} 21$ were increased after $10 \mathrm{~Gy}$ IR.

Furthermore, expression levels of p53 and p21 were enhanced in cells treated with a combination of $1 \mu \mathrm{M}$ Naph and 10 Gy IR. On the contrary, the expression levels of DNMT1, UHRF1 and HDAC1 were decreased after 10 Gy of irradiation. The decreased expression levels of DNMT1, UHRF1 and HDAC1 were further reduced in cells treated with a combination of $1 \mu \mathrm{M}$ Naph and 10 Gy IR (Fig. 2C). These data suggest that Naph is a potential radiosensitizer that increases the sensitivity to IR-induced cell death in breast cancer.

DNMT1/UHRF1/HDAC1 complex and p53 are reciprocally localized on the 21 promoter in MCF-7 cells under treatment with Naph and IR. UHRF1 recruits transcriptional repressors DNMT1 and HDAC1 through its distinct domains. Moreover, it is known that UHRF1 recruits and cooperates with DNMT1 and HDAC1 on the promoter of $\mathrm{p} 21$, thereby inhibiting the expression of p21 (31-36). In order to investigate the occupancy of DNMT1/UHRF1/HDAC1 and p53 on the p21 promoter under various conditions such as Naph, IR and NaphsIR treatments, we performed the chromatin immunoprecipitation (ChIP) assay. As expected, binding of DNMT1, UHRF1 and HDAC1 to the p21 promoter after IR and Naph treatment gradually decreased when compared to that under untreated conditions (Fig. 3A-C). In contrast, the occupancy of p53 on 

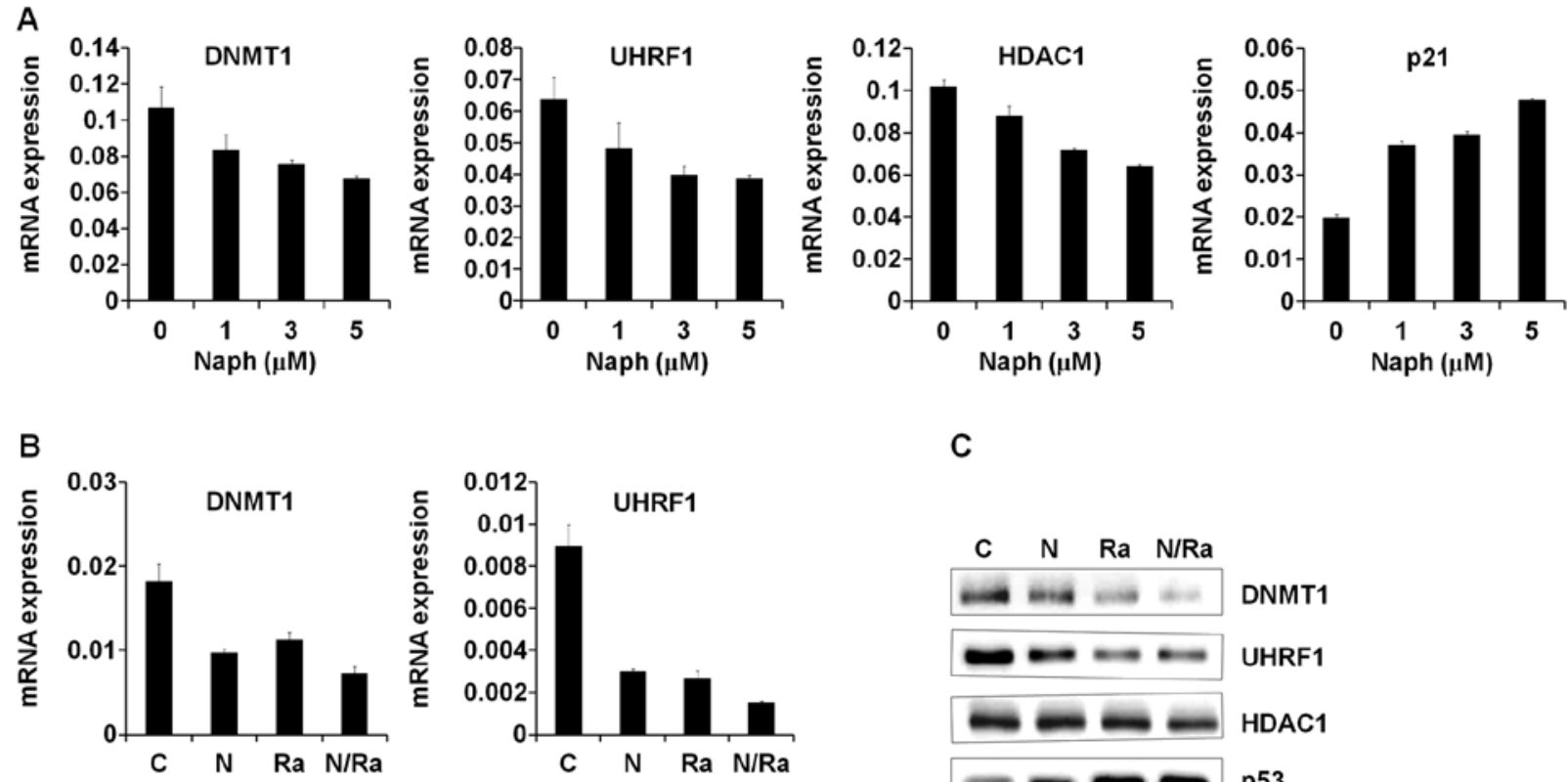

C
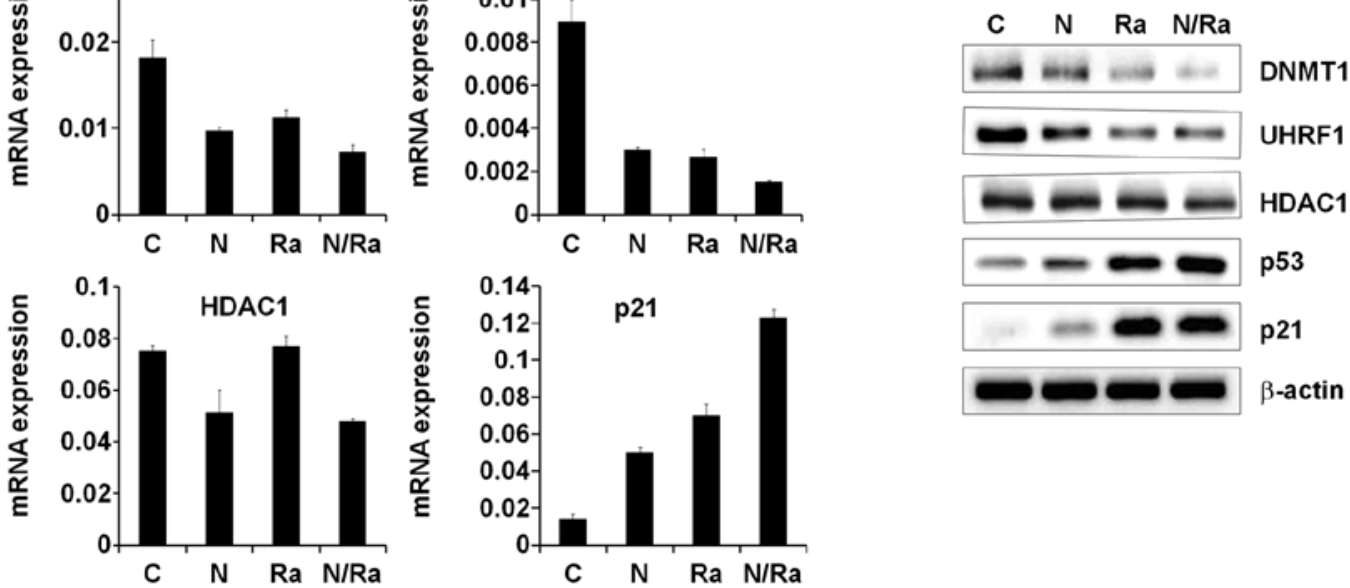

Figure 2. Expression of p21 and DNMT1/UHRF1/HDAC1 in MCF-7 cells after treatment with Naph and/or IR. (A) DNMT1, UHRF1, HDAC1 and p21 mRNA expression patterns in MCF-7 cells under 1, 3 and $5 \mu \mathrm{M}$. Naph conditions were assessed by quantitative RT-PCR. C, untreated condition; N1, naphthazarin $(1 \mu \mathrm{M})$; N3, naphthazarin $(3 \mu \mathrm{M})$; N5, naphthazarin $(5 \mu \mathrm{M})$. (B) DNMT1, UHRF1, HDAC1 and p21 mRNA expression patterns in MCF-7 cells treated with $1 \mu \mathrm{M}$ Naph, IR and/or combined conditions assessed by quantitative RT-PCR. Results represent mRNA levels normalized to the levels of GAPDH mRNA. Relative DNMT1, UHRF1, HDAC1 and p21 mRNA levels are shown as mean \pm standard deviation of three independent experiments. C, untreated condition; $\mathrm{N}$, naphthazarin $(1 \mu \mathrm{M})$; R, ionizing radiation (IR, $10 \mathrm{~Gy})$; NR, Naph $(1 \mu \mathrm{M})+\mathrm{IR}(10 \mathrm{~Gy})$. (C) Western blot analyses of DNMT1, UHRF1, HDAC1, p53 and p21 protein expression patterns in MCF-7 cells treated with Naph $(1 \mu \mathrm{M})$ and IR and/or combined conditions.
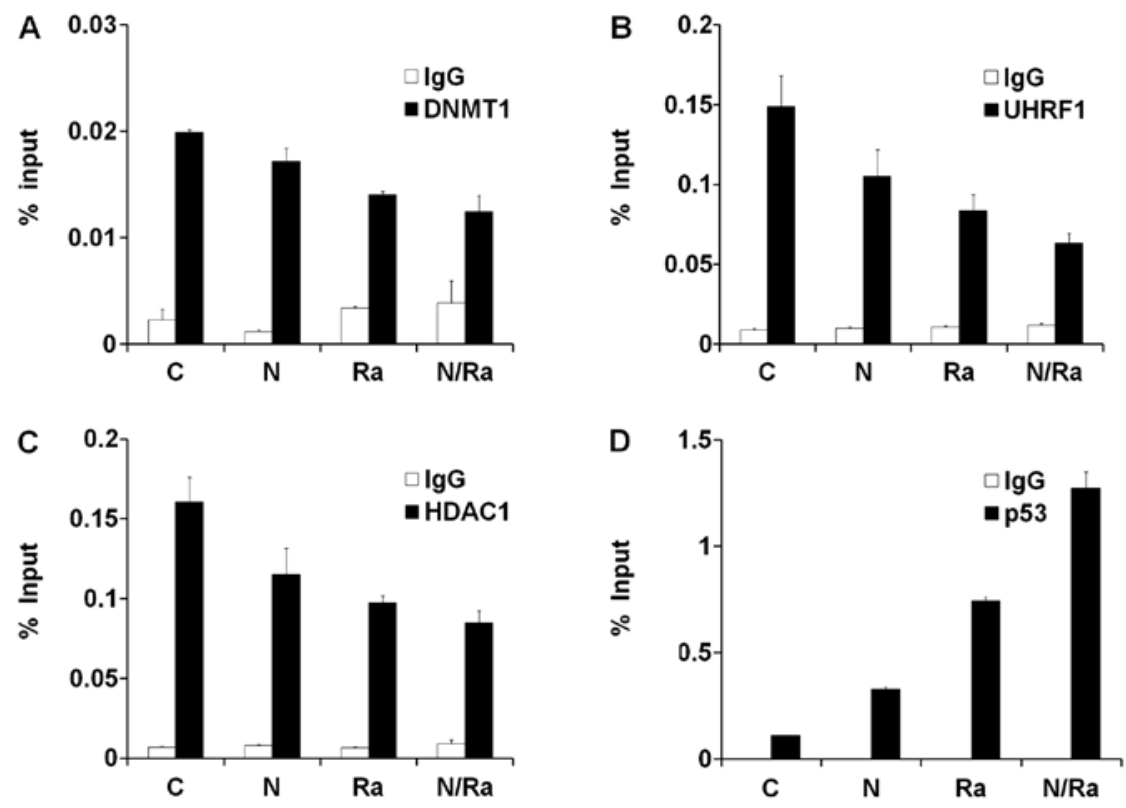

Figure 3. Localization of DNMT1/UHRF1/HDAC1 complex and p53 on the p21 promoter in MCF-7 cells after treatment with Naph and IR. Chromatin immunoprecipitation (ChIP) assay for DNMT1 (A), UHRF1 (B), HDAC1 (C) and p53 (D) at the p21 gene promoter region in MCF-7 cells under $1 \mu$ M Naph, $10 \mathrm{~Gy}$ IR, and/or combined conditions. Cross-linked and sheared chromatin was immunoprecipitated with the anti-DNMT1 antibody (white bar, left upper panel), the anti-UHRF1 antibody (white bar, right upper panel), the anti-HDAC1 antibody (white bar, left bottom panel), the anti-p53 antibody (white bar, right bottom panel), and the anti-IgG antibody (black bar). The results are shown as a percentage of the chromatin input. ChIP samples were quantified by RT-PCR. Data represent mean \pm standard deviation of triplicates. Representative data from three independent experiments. 
A

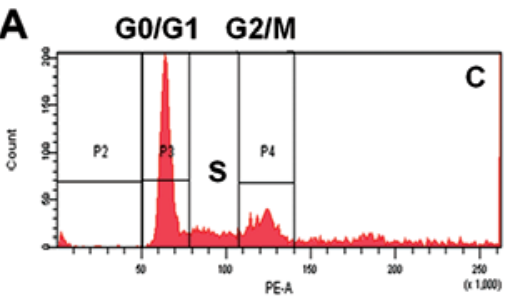

G0/G1 G2/M

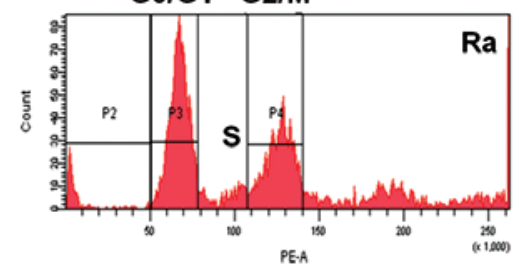

G0/G1 G2/M
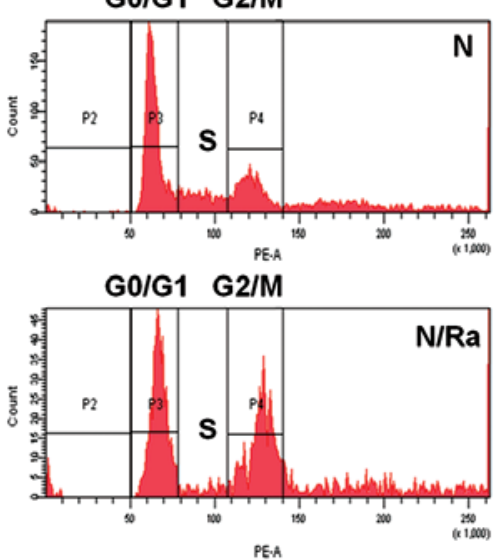

B

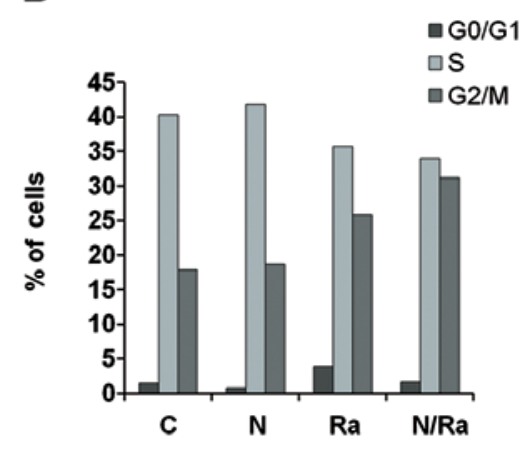

C

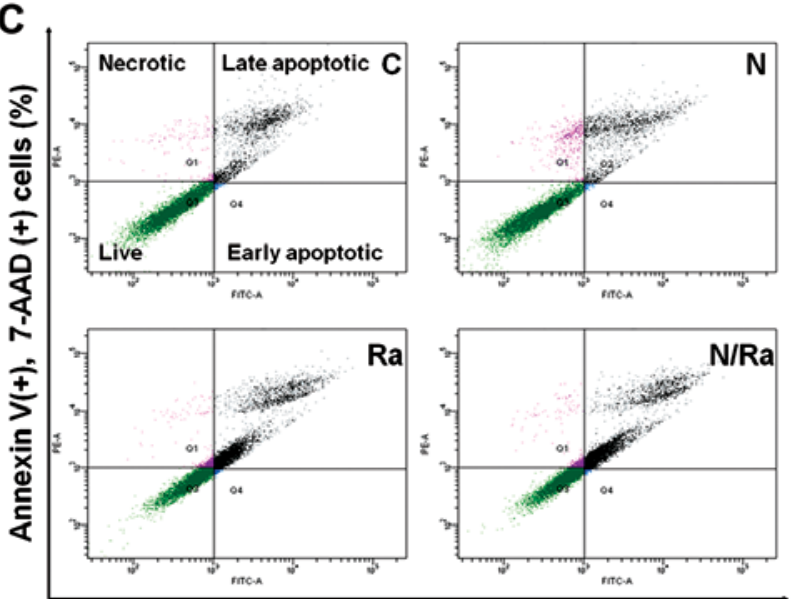

D

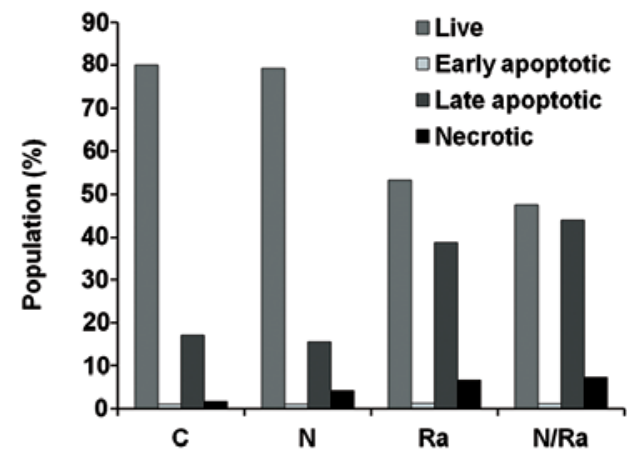

Annexin V(+), 7-AAD(-) cells (\%)

Figure 4. Effect of Naph and IR on cell cycle and apoptosis distribution in MCF-7 cells. (A) Cell cycle analysis of MCF-7 cells treated with $1 \mu$ M Naph, $10 \mathrm{~Gy} \mathrm{IR}$ and/or combined conditions for $48 \mathrm{~h}$ on FACSAria. (B) The cell cycle results of indicated samples were expressed through the quantification graph. (C) Apoptosis detection assay was performed using the Annexin V and 7-AAD double staining assay in MCF-7 cells. MCF-7 cells were treated with Naph and/or IR for $48 \mathrm{~h}$, stained with Annexin V and 7-AAD and analyzed on FACSAria. (D) Quantification graph of Annexin V and 7-AAD assay obtained from FACSAria.

the p21 promoter dramatically increased after combinatorial treatment of IR and Naph (Fig. 3D). Taken together, these results demonstrate that dissociation of DNMT1/UHRF1/ HDAC1 from the p21 promoter during treatment with Naph and IR enhances the recruitment of p53 to the p21 promoter, thereby activating the transcription of p21 in MCF-7 cells.

Combination of Naph and IR induces G2/M cell cycle arrest and apoptosis in $M C F-7$ cells. Since we identified that mRNA and protein levels of cell cycle related p53 and p 21 are regulated by Naph and/or IR, we next investigated whether Naph and IR induce cell cycle arrest in the MCF-7 cells using flow cytometry (FACSAria). In controls, flow cytometry analysis showed $1.5 \%$ of cells in G0/G1 phase, $40.3 \%$ of cells in S phase, and $17.9 \%$ of cells in $\mathrm{G} 2 / \mathrm{M}$ phase. In contrast, in cells treated with $1 \mu \mathrm{M}$ Naph, the proportions of cells in G0/G1, S, and G2/M phases were $0.8,41.8$ and $18.7 \%$, respectively, and the proportions of cells exposed to 10 Gy IR in G0/G1, S, and G2/M phases were $3.9,35.7$ and $25.8 \%$, respectively. When cells were treated with $1 \mu \mathrm{M}$ Naph and IR, the proportions of cells in G0/G1, S, and G2/M phases were 1.6, 34.0 and $31.2 \%$, respectively. These results suggest that combinatorial treatment of Naph and IR induces $\mathrm{G} 2 / \mathrm{M}$ phase arrest compared with single treatment (Fig. 4A and B).

Next, we investigated whether Naph and IR induce MCF-7 cell apoptosis using the Annexin V/7-AAD double staining kit. The combinatorial effect of Naph and IR was evaluated after $48 \mathrm{~h}$ of treatment. Cells negative for 7-AAD and positive for PE Annexin $\mathrm{V}$ were regarded as early apoptotic cells (Annexin $\mathrm{V}^{+}, 7-\mathrm{AAD}^{-}$); 7-AAD and Annexin $\mathrm{V}$ positive cells were defined as late apoptotic cells (Annexin $\mathrm{V}^{+}$, 7-AAD ${ }^{+}$); 7-AAD positive and PE Annexin $\mathrm{V}$ negative cells were considered as necrotic cells (Annexin $\mathrm{V}^{-}, 7-\mathrm{AAD}^{+}$). The combined treatment of $1 \mu \mathrm{M}$ Naph and 10 Gy IR in MCF-7 cells significantly increased the apoptotic effect compared with single treatment of IR or Naph. Increased number of late apoptotic cells were observed in the combined treatment group (43.9\%) compared with the Naph alone group (15.6\%) and IR alone group (38.7\%). However, the cells treated with Naph and IR showed a slightly increased necrotic portion (7.3\%) and there was a moderate decrease in the number of live cells $(47.5 \%)$ in comparison with cells treated with Naph or IR treatment alone (Fig. 4C and D). These data suggest that combinatorial treatment of Naph and IR has a potential 

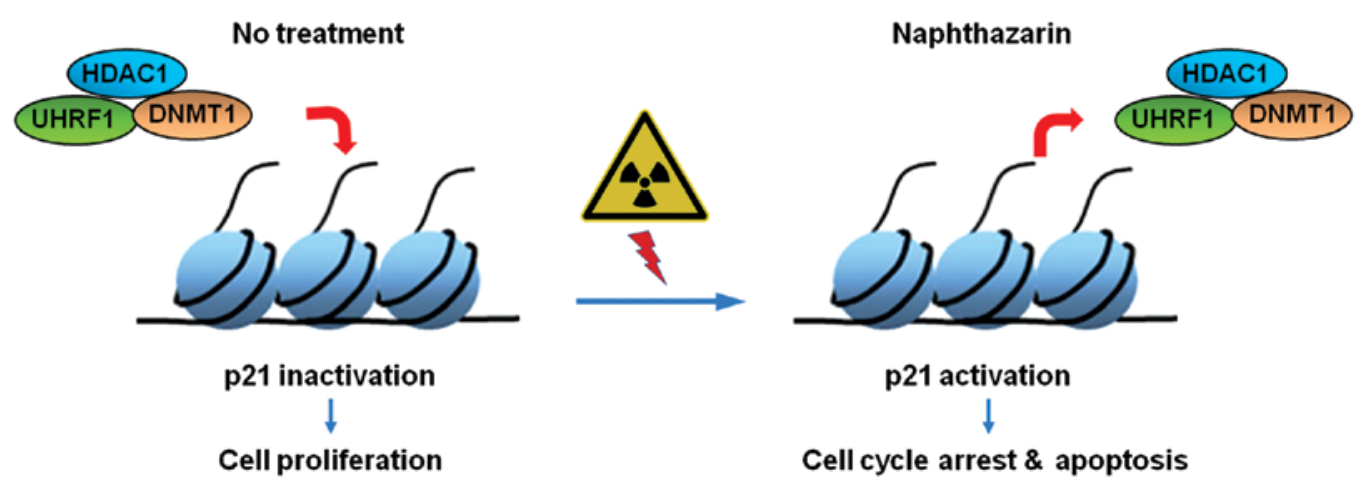

Figure 5. Model describing the effect of Naph as a radiosensitizer via p21 gene activation and DNMT1/UHRF1//HDAC1 dissociation in Naph- and IR-treated MCF-7 cells.

synergistic effect on the regulation of cell cycle arrest and apoptosis in MCF-7 cells.

\section{Discussion}

Radiotherapy is used in over $50 \%$ of patients during the course of cancer treatment both as a curative modality and for palliation. However, radioresistance and toxic side effects impeding dose escalation are major obstacles to the success of radiation therapy $(39,40)$. Therefore, there is a critical need for the discovery of a novel radiosensitizer that can improve the efficacy of radiotherapy. Several studies have reported that plant-derived natural products have cancer chemopreventive and chemotherapeutic properties. The use of natural products as antitumor agents or radiosensitizers for the management of human cancers is an attractive idea because they are readily available and exhibit little or no toxicity (3-11).

Naph is a natural component of the roots of several members of the genus Boraginaceae and is well-known to have antitumor cytotoxic effects in cancer cells (12-16). However, the biological role and mechanism of cytotoxicity and the specific molecular target of Naph in cancer cells are yet to be established. In this study, we demonstrated the role and mechanism of Naph as an effective radiosensitizer and adjuvant therapy in breast cancer. We first evaluated the effect of Naph and IR on the proliferation of MCF-7 breast cancer cells. The results showed that the growth of MCF-7 cells treated with Naph was decreased in a concentration-dependent manner (Fig. 1A).

To diminish the toxic side effect of Naph, we used a low dose $(1 \mu \mathrm{M})$ of Naph for further experiments to observe its synergistic effect with radiation. Indeed, we observed that the viability of cells treated with a low concentration $(1 \mu \mathrm{M})$ of Naph and IR of 10 Gy was decreased more than that of MCF-7 cells treated with single Naph and 10 Gy of IR. We also demonstrated that the cells treated with Naph and IR of 10 Gy showed morphologic changes and less number of the prominent cells than untreated cells (Fig. 1C). Therefore, these results suggest that combinational treatment of Naph and IR more effectively inhibits cancer cell viability and proliferation than a single treatment.

Several studies have reported that DNMT1/UHRF1/ HDAC1 complex negatively regulates the expression of $\mathrm{p} 21$, which inhibits cell proliferation (31-36). To determine the mechanism of antitumor cytotoxicity and the specific molecular target of Naph, we next examined the expression of p21 and its regulatory factors (DNMT1/UHRF1/HDAC1). Our results of Real-time PCR showed that Naph downregulated DNMT1, UHRF1, and HDAC1 mRNA expressions in a dose-dependent manner (Fig. 2A). Moreover, we identified that the expression level of p21 was dramatically upregulated by co-treatment of Naph and IR, whereas the expression levels of DNMT1, UHRF1, and HDAC1 were downregulated (Fig. 2B and C).

It is well documented that $\mathrm{p} 21$ is a downstream target of p53, which regulates transcription, DNA repair, cell cycle arrest, differentiation, senescence, genomic instability, apoptosis, and survival as well as glucose metabolism, oxidative stress, and angiogenesis (31-34). Therefore, we measured the protein levels of p53 and p21 together with levels of repressive factors, including DNMT1, UHRF1, and HDAC1. Our results showed that the expression levels of p53 and its downstream p21 were enhanced in cells co-treated with $1 \mu \mathrm{M}$ Naph and 10 Gy IR compared to Naph or IR alone treated cells (Fig. 2C). These results support that Naph increases the sensitivity to IR-induced cell cycle arrest or apoptosis through the p53-p21 pathway in breast cancer. In order to identify the regulatory mechanism of p21 expression, we performed the ChIP analysis. Our ChIP results revealed that the repressive factors, DNMT1/ UHRF1/HDAC1 were dissociated from the p21 promoter by treatment with Naph and IR compared with that under untreated conditions. On the contrary, the occupancy of p53 on the p21 promoter was significantly increased after combinational treatment of Naph and IR (Fig. 3). Therefore, these results demonstrate that DNMT1/UHRF1/HDAC1 complex and p53 are reciprocally localized on the p21 promoter under treatment with Naph and IR, thereby regulating the transcription of p21 in MCF-7 cells.

Deregulation of the cell cycle and apoptosis are frequent occurrences in cancer development. In this study, p53 and its target gene, p21, were dependently regulated by Naph and/or IR. We investigated whether Naph and IR induce cell cycle arrest in the MCF-7 cells using flow cytometry. The cells treated with $1 \mu \mathrm{M}$ Naph or $10 \mathrm{~Gy}$ IR alone showed a slight increase in the proportion of cells in G2/M phase compared with the untreated cells. However, the cells treated with $1 \mu \mathrm{M}$ Naph and 10 Gy IR together showed a significant increase in the proportion of cells in $\mathrm{G} 2 / \mathrm{M}$ phase compared with the cells treated with Naph and IR alone (Fig. 4A and B). These results suggest that Naph and IR induce G2/M phase arrest. Moreover, 
the radiosensitizing effect of Naph was assessed through the apoptosis assay. Our data showed that combined treatment of $1 \mu \mathrm{M}$ Naph and $10 \mathrm{~Gy}$ IR in MCF-7 cells significantly increased the apoptotic effect compared with single treatment of IR or Naph (Fig. 4C and D).

In conclusion, our findings show that combinational treatment of Naph and IR dramatically inhibits cell proliferation. Furthermore, the dissociation of DNMT1/UHRF1/HDAC1 from the p21 promoter during treatment with Naph and IR enhances the recruitment of p53 to the p21 promoter, thereby inducing cell cycle arrest and apoptosis in MCF-7 cells. These findings lead us to believe that Naph might be a potential radiosensitizer in breast cancer (Fig. 5).

\section{Acknowledgements}

This study was supported by the National R\&D Program through the Dongnam Institute of Radiological \& Medical Sciences (DIRAMS) (50595-2014) and the [Basic Science Research Program] through the National Research Foundation of Korea (NRF) (NRF-2014 M2A2A 7043665) funded by the Ministry of Science, ICT and Future Planning.

\section{References}

1. Jemal A, Bray F, Center MM, Ferlay J, Ward E and Forman D: Global cancer statistics. CA Cancer J Clin 61: 69-90, 2011.

2. Bozovic-Spasojevic I, Azambuja E, McCaskill-Stevens W, Dinh P and Cardoso F: Chemoprevention for breast cancer. Cancer Treat Rev 38: 329-339, 2012.

3. Zhang Y, Luo M, Zu Y, et al: Dryofragin, a phloroglucinol derivative, induces apoptosis in human breast cancer MCF-7 cells through ROS-mediated mitochondrial pathway. Chem Biol Interact 199: 129-136, 2012.

4. Krifa M, Pizzi A, Mousli M, Chekir-Ghedira L, Leloup L and Ghedira K: Limoniastrum guyonianum aqueous gall extract induces apoptosis in colorectal cancer cells by inhibiting calpain activity. Tumour Biol 35: 7877-7885, 2014.

5. Ziech D, Anestopoulos I, Hanafi R, et al: Pleiotrophic effects of natural products in ROS-induced carcinogenesis: The role of plant-derived natural products in oral cancer chemoprevention. Cancer Lett 327: 16-25, 2012.

6. Magee PJ, Owusu-Apenten R, McCann MJ, Gill CI and Rowland IR: Chickpea (Cicer arietinum) and other plant-derived protease inhibitor concentrates inhibit breast and prostate cancer cell proliferation in vitro. Nutr Cancer 64: 741-748, 2012.

7. Fang Y, DeMarco VG and Nicholl MB: Resveratrol enhances radiation sensitivity in prostate cancer by inhibiting cell proliferation and promoting cell senescence and apoptosis. Cancer Sci 103: 1090-1098, 2012

8. Wang Q, Fan H, Liu Y, et al: Curcumin enhances the radiosensitivity in nasopharyngeal carcinoma cells involving the reversal of differentially expressed long non-coding RNAs. Int J Oncol 44: 858-864, 2014.

9. Cragg GM and Newman DJ: Plants as a source of anti-cancer agents. J Ethnopharmacol 100: 72-79, 2005.

10. Srivastava V, Negi AS, Kumar JK, Gupta MM and Khanuja SP Plant-based anticancer molecules: A chemical and biological profile of some important leads. Bioorg Med Chem 13: 5892-5908, 2005.

11. Kourounakis AP, Assimopoulou AN, Papageorgiou VP, Gavalas A and Kourounakis PN: Alkannin and shikonin: Effect on free radical processes and on inflammation - a preliminary pharmacochemical investigation. Arch Pharm (Weinheim) 335: 262-266, 2002

12. Acharya BR, Bhattacharyya S, Choudhury D and Chakrabarti G: The microtubule depolymerizing agent naphthazarin induces both apoptosis and autophagy in A549 lung cancer cells. Apoptosis 16: 924-939, 2011.

13. Kundaković T, Stanojković T, Juranić $\mathrm{Z}$ and Kovacević N: Cytotoxicity in vitro of naphthazarin derivatives from onosma arenaria. Phytother Res 20: 602-604, 2006.
14. Johansson AC, Norberg-Spaak L and Roberg K: Role of lysosomal cathepsins in naphthazarin- and fas-induced apoptosis in oral squamous cell carcinoma cells. Acta Otolaryngol 126: 70-81, 2006.

15. Song GY, Kim Y, You YJ, Cho H, Kim SH, Sok DE and Ahn BZ: Naphthazarin derivatives (VI): Synthesis, inhibitory effect on DNA topoisomerase-I and antiproliferative activity of 2- or 6-(1-oxyiminoalkyl)-5,8-dimethoxy-1,4-naphthoquinones. Arch Pharm (Weinheim) 333: 87-92, 2000.

16. Kim JA, Lee EK, Park SJ, Kim ND, Hyun DH, Lee CG, Lee JH, Yang KM, Heo K and Son TG: Novel anti-cancer role of naphthazarin in human gastric cancer cells. Int J Oncol 40: 157-162, 2012.

17. Sherr CJ and Roberts JM: CDK inhibitors: Positive and negative regulators of G1-phase progression. Genes Dev 13: 1501-1512, 1999.

18. Lim S and Kaldis P: Cdks, cyclins and CKIs: Roles beyond cell cycle regulation. Development 140: 3079-3093, 2013.

19. Lee YH and Bae YS: Phospholipase D2 downregulation induces cellular senescence through a reactive oxygen species-p53p21Cip1/WAF1 pathway. FEBS Lett 588: 3251-3258, 2014.

20. Saha K, Adhikary G, Kanade SR, Rorke EA and Eckert RL: p38 regulates p53 to control p21Cip1 expression in human epidermal keratinocytes. J Biol Chem 289: 11443-11453, 2014.

21. Yadav V, Sultana S, Yadav J and Saini N: Gatifloxacin induces $\mathrm{S}$ and G2-phase cell cycle arrest in pancreatic cancer cells via p21/p27/p53. PLoS One 7: e47796, 2012.

22. Khan M, Rasul A, Yi F, Zhong L and Ma T: Jaceosidin induces p53-dependent G2/M phase arrest in U87 glioblastoma cells. Asian Pac J Cancer Prev 12: 3235-3238, 2011.

23. Powell BL, van Staveren IL, Roosken P, Grieu F, Berns EM and Iacopetta B: Associations between common polymorphisms in TP53 and p21WAF1/Cip1 and phenotypic features of breast cancer. Carcinogenesis 23: 311-315, 2002.

24. Darcy KM, Brady WE, McBroom JW, et al: Associations between p53 overexpression and multiple measures of clinical outcome in high-risk, early stage or suboptimally-resected, advanced stage epithelial ovarian cancers A Gynecologic Oncology Group study. Gynecol Oncol 111: 487-495, 2008.

25. Unoki M, Nishidate T and Nakamura Y: ICBP90, an E2F-1 target, recruits HDAC1 and binds to methyl-CpG through its SRA domain. Oncogene 23: 7601-7610, 2004

26. Bostick M, Kim JK, Estève PO, Clark A, Pradhan S and Jacobsen SE: UHRF1 plays a role in maintaining DNA methylation in mammalian cells. Science 317: 1760-1764, 2007.

27. Achour M, Jacq X, Rondé P, et al: The interaction of the SRA domain of ICBP90 with a novel domain of DNMT1 is involved in the regulation of VEGF gene expression. Oncogene 27: 2187-2197, 2008

28. Achour M, Fuhrmann G, Alhosin M, et al: UHRF1 recruits the histone acetyltransferase Tip60 and controls its expression and activity. Biochem Biophys Res Commun 390: 523-528, 2009.

29. Alhosin M, SharifT, Mousli M,Etienne-Selloum N, Fuhrmann G, Schini-Kerth VB and Bronner C: Down-regulation of UHRF1, associated with re-expression of tumor suppressor genes, is a common feature of natural compounds exhibiting anti-cancer properties. J Exp Clin Cancer Res 30: 41, 2011.

30. Bronner C, Achour M, Arima Y, Chataigneau T, Saya H and Schini-Kerth VB: The UHRF family: Oncogenes that are drugable targets for cancer therapy in the near future. Pharmacol Ther 115: 419-434, 2007.

31. Unoki M, Brunet J and Mousli M: Drug discovery targeting epigenetic codes: The great potential of UHRF1, which links DNA methylation and histone modifications, as a drug target in cancers and toxoplasmosis. Biochem Pharmacol 78: 1279-1288, 2009.

32. Kim JK, Estève PO, Jacobsen SE and Pradhan S: UHRF1 binds G9a and participates in p21 transcriptional regulation in mammalian cells. Nucleic Acids Res 37: 493-505, 2009.

33. Yamaguchi T, Cubizolles F, Zhang Y, Reichert N, Kohler H, Seiser C and Matthias P: Histone deacetylases 1 and 2 act in concert to promote the G1-to-S progression. Genes Dev 24: 455-469, 2010.

34. Saldanha SN, Kala R and Tollefsbol TO: Molecular mechanisms for inhibition of colon cancer cells by combined epigeneticmodulating epigallocatechin gallate and sodium butyrate. Exp Cell Res 324: 40-53, 2014.

35. Tien AL, Senbanerjee S, Kulkarni A, et al: UHRF1 depletion causes a G2/M arrest, activation of DNA damage response and apoptosis. Biochem J 435: 175-185, 2011. 
36. Vijayaraghavalu S, Dermawan JK, Cheriyath V and Labhasetwar V: Highly synergistic effect of sequential treatment with epigenetic and anticancer drugs to overcome drug resistance in breast cancer cells is mediated via activation of p21 gene expression leading to G2/M cycle arrest. Mol Pharm 10: 337-352, 2013.

37. Zhang X, Li X, Zhang N, Yang Q and Moran MS: Low doses ionizing radiation enhances the invasiveness of breast cancer cells by inducing epithelial-mesenchymal transition. Biochem Biophys Res Commun 412: 188-192, 2011.

38. Tsai JH, Hsu LS, Lin CL, Hong HM, Pan MH, Way TD and Chen WJ: 3,5,4'-Trimethoxystilbene, a natural methoxylated analog of resveratrol, inhibits breast cancer cell invasiveness by downregulation of PI3K/Akt and Wnt/ $\beta$-catenin signaling cascades and reversal of epithelial-mesenchymal transition. Toxicol Appl Pharmacol 272: 746-756, 2013.
39. Luo H, Wang L, Schulte BA, Yang A, Tang S and Wang GY: Resveratrol enhances ionizing radiation-induced premature senescence in lung cancer cells. Int J Oncol 43: 1999-2006, 2013.

40. Hall EJ and Wuu CS: Radiation-induced second cancers: The impact of 3D-CRT and IMRT. Int J Radiat Oncol Biol Phys 56: 83-88, 2003. 